\title{
A New Wideband Mutual Coupling Compensation Method for Adaptive Arrays Based on Element Pattern Reconstruction
}

\author{
Qiulin Huang, Feng Wei, Lihua Yuan, Hongxing Zhou, and Xiaowei Shi \\ Science and Technology on Antenna and Microwave Laboratory, Xidian University, Xian 710071, China \\ Correspondence should be addressed to Qiulin Huang; qiulhuang@mail.xidian.edu.cn
}

Received 19 November 2013; Revised 10 January 2014; Accepted 13 January 2014; Published 20 February 2014

Academic Editor: Sembiam R. Rengarajan

Copyright ( 2014 Qiulin Huang et al. This is an open access article distributed under the Creative Commons Attribution License, which permits unrestricted use, distribution, and reproduction in any medium, provided the original work is properly cited.

\begin{abstract}
A new mutual coupling compensation method for wideband adaptive arrays is proposed. The new method is developed by combining the element pattern reconstruction method and the system identification method. The element pattern reconstruction method is valid and effective in the mutual coupling compensation for adaptive arrays such as dipole arrays and microstrip arrays. Each entry of the wideband compensation matrix is represented as an analytical expression against frequency. The polynomial coefficients and orders of all entries are obtained via the system identification method. The new wideband compensation method is characterized by the good adaptability of element structures and polarizations owing to the advantages of element pattern reconstruction method. A wideband microstrip array is designed to test the validity and effectiveness of the wideband compensation method.
\end{abstract}

\section{Introduction}

At present, wideband antenna arrays are widely used in the adaptive signal processing systems, and microstrip antennas are often employed in these systems. However, the performance of adaptive array signal processing is seriously influenced by the mutual coupling effect of arrays, especially in the lower frequency band. It can be explained via the relationship between the frequency and electric length. Over the past 30 years, various mutual coupling compensation methods have been proposed, such as the open circuit voltage method, the receiving mutual impedance method, the minimum norm method, the method proposed by Su et al., and the method introduced by Yuan et al. [1-8]. In most of the methods, the compensation matrices are obtained through the analysis of current distributions or induced terminal voltages on antenna arrays. The open circuit scattering of arrays was not taken into account in the open circuit voltage method, which degrades the compensation effect of this method [1]. The receiving mutual impedance method and minimum norm method can provide more accurate mutual coupling compensations owing to the improvements in processing the scattering effect [2-4]. The pattern of ideal point source model was used in the method proposed by
Su et al. [5-7]. For the antenna array composed of dipoles or monopoles, $\mathrm{H}$-plane pattern of the element is isotropic, which is the same with the ideal point source. In this case, Su's method can provide accurate mutual coupling compensation for the incident signals in the H-plane. However, methods proposed in [2-7] are more suitable for antenna arrays composed of wire elements such as dipoles and monopoles. For arrays composed of planar elements such as microstrip antennas, it is difficult to find a proper compensation matrix to match the incident signals coming from various directions by using the compensation methods mentioned above. In the method proposed by Yuan et al., the universal steering vector was used in DOA estimations so that the received voltages of the array can be used directly to find directions without calibration [8]. The universal steering vector is composed of the embedded element patterns at given direction. However, the compensation matrix was given through the relationship between the universal steering vector and the conventional steering vector due to the ideal source model, and the universal steering vector was related with the incident angles and polarization of the incident signals, which limits the applications of this method in adaptive antenna arrays. Although the compensation matrix independent of incident angles was obtained in [9-11], the compensation 
matrix also described the relationship between the universal steering vector and the conventional steering vector. It is expected that applications of ideal source model in the above compensation methods cannot guarantee the decoupling of received signals. Actually, residual mutual coupling effects still remain when the ideal source model is employed in the compensation method, since the received signal due to the isolated antennas is uninfluenced by the mutual coupling.

The joint estimation of direction-of-arrivals (DOAs) and calibration matrix provided a feasible approach for the mutual coupling calibration of microstrip arrays [12-14]. However, joint estimation methods are usually used for uniform linear arrays (ULAs) and uniform circular arrays (UCAs). The number of mutual coupling parameters can be decreased for regular array structures, since the calibration matrix is characterized by the complex Toeplitz structure, which is important for solving the joint estimation problems. More importantly, the time consumption of joint estimations is difficult to satisfy the real-time requirement in many practical applications.

In the literature, a wideband mutual coupling compensation method for a dipole array was investigated, and the system identification method was employed to obtain the wideband calibration matrix [15-17]. It was verified that the system identification method was effective in calculating the wideband compensation matrix. However, the system identification method proposed above was based on the receiving mutual impedance method that is more suitable for the arrays composed of wire elements, thus limiting the applications of this wideband compensation method.

Recently, the element pattern reconstruction method was proposed by the authors to compensate for the mutual coupling effect of adaptive arrays $[18,19]$. This method was developed on the basis of the relationship between the embedded element patterns and isolated element patterns of an array. It can be used not only in arrays composed of wire elements, but also in arrays composed of planar elements such as microstrip antennas. Additionally, this method is suitable for linearly polarized arrays as well as circularly polarized arrays. It is important that the compensation matrix is independent of the incident angle and polarization of signals. The good performance of the method was verified with a dipole array, linearly polarized microstrip array, circularly polarized microstrip array, and conformal microstrip array. Based on this consideration, the element pattern reconstruction method has the potential to develop more effective compensation methods for the wideband adaptive arrays.

In this paper, the combination of the element pattern reconstruction method and system identification method is proposed for the wideband mutual coupling compensation. The new method has a good adaptability for the element structures and polarizations owing to the characteristics of the element pattern reconstruction method. The new method can be employed in the wideband frequency-hopping mode and for proper wideband signals. A wideband microstrip array with five elements is designed to verify the effectiveness of the new wideband compensation method.

\section{Theory}

2.1. Element Pattern Reconstruction Method. It is known that an antenna pattern corresponds with the magnitude of the received signals in the given directions. In addition, the pattern of an element in the isolated state with other elements removed from the array is not influenced by the mutual coupling, while the pattern of an embedded element will be influenced by mutual coupling. The above considerations provide us with an approach to compensate for the mutual coupling of adaptive arrays via the transformation of element patterns. The element pattern reconstruction method is based on the fact that when all embedded element patterns are transformed to coincide with the corresponding isolated element patterns in a certain direction, the received signals owing to this direction would be decoupled after being transformed in the same way. The compensation matrix is obtained through the transformation of the element patterns in the proposed method, which is different with existing methods.

Generally, the main polarization component and the cross polarization component are seen by an antenna simultaneously. For the linearly polarized antenna, the main polarization component is often the $\theta$ or $\varphi$ component of the electric field. For circularly polarized antennas, the main polarization component is the electric field of the left hand circular polarization (LHCP) or the right hand circular polarization (RHCP) components. In a well-designed microstrip antenna, the main polarization component contributes the dominant part in the total electric field. Consequently, the main polarization component plays a dominant role in the mutual coupling calibrations. The main polarization component is thus considered for the element pattern reconstruction method.

Consider an antenna array with $N$ elements with each element terminated in a load $Z_{L}$. Two kinds of element patterns are involved in the element pattern reconstruction method. One is the embedded element pattern that is calculated with other elements terminated with a load. Another is the isolated element pattern that is calculated with other elements removed from the array. A relationship between two kinds of patterns is established so as to transform the embedded element patterns into the isolated element patterns; that is,

$$
\left[\begin{array}{c}
E_{1}^{i}(\theta, \varphi) \\
E_{2}^{i}(\theta, \varphi) \\
\vdots \\
E_{N}^{i}(\theta, \varphi)
\end{array}\right]=\mathbf{C}\left[\begin{array}{c}
E_{1}(\theta, \varphi) \\
E_{2}(\theta, \varphi) \\
\vdots \\
E_{N}(\theta, \varphi)
\end{array}\right],
$$

where $E_{n}^{i}(\theta, \varphi)$ and $E_{n}(\theta, \varphi)$ with $n=1,2, \ldots, N$ represent the element pattern in the isolated state and in the embedded state, respectively. Matrix $\mathbf{C}$ represents the compensation matrix which can be used to compensate for the mutual coupling effect of the array. It is to be noted that both magnitude and phase of electric fields are taken into account in (1). In order to accurately calculate the compensation matrix $\mathbf{C}$ that can adapt to wide angle range, multiple 
directions should be sampled from the element patterns. Assume that $M$ directions are chosen; one obtains

$$
\begin{gathered}
{\left[\begin{array}{c}
E_{1}^{i}\left(\theta_{1}, \varphi_{1}\right) \cdots E_{1}^{i}\left(\theta_{M}, \varphi_{M}\right) \\
E_{2}^{i}\left(\theta_{1}, \varphi_{1}\right) \cdots E_{2}^{i}\left(\theta_{M}, \varphi_{M}\right) \\
\vdots \\
E_{N}^{i}\left(\theta_{1}, \varphi_{1}\right) \cdots E_{N}^{i}\left(\theta_{M}, \varphi_{M}\right)
\end{array}\right]} \\
=\mathbf{C}\left[\begin{array}{c}
E_{1}\left(\theta_{1}, \varphi_{1}\right) \cdots E_{1}\left(\theta_{M}, \varphi_{M}\right) \\
E_{2}\left(\theta_{1}, \varphi_{1}\right) \cdots E_{2}\left(\theta_{M}, \varphi_{M}\right) \\
\vdots \\
E_{N}\left(\theta_{1}, \varphi_{1}\right) \cdots E_{N}\left(\theta_{M}, \varphi_{M}\right)
\end{array}\right] .
\end{gathered}
$$

Consequently, the least square solution that satisfies

$$
\min \left\|\mathbf{C E}-\mathbf{E}^{i}\right\|
$$

can be obtained; that is,

$$
\mathbf{C}=\mathbf{E}^{i} \mathbf{E}^{H}\left(\mathbf{E E}^{H}\right)^{-1}
$$

where $\mathbf{E}^{i}$ represents the electric field matrix owing to the isolated elements and $\mathbf{E}$ the electric field matrix owing to the embedded elements. Superscript $H$ denotes the Hermitian transpose operation and operator $\|\cdot\|$ denotes the Frobenius norm of a matrix. For matrix $\mathbf{C}$ with the dimension of $N \times M$, the Frobenius norm is written as

$$
\|\mathbf{C}\|=\left\{\sum_{n=1}^{N} \sum_{m=1}^{M}\left|c_{n m}\right|^{2}\right\}^{1 / 2}
$$

where $c_{n m}$ is an entry of matrix $\mathbf{C}$.

A compensation matrix can be calculated via (4). This solution is valid for the sampled directions. It is known that slow continuous change of element pattern exists in the main lobe and especially in the $3 \mathrm{~dB}$ beamwidth. It is thus possible to obtain a compensation matrix that is valid for the $3 \mathrm{~dB}$ beamwidth or a bit larger angle range. In nature, the compensation matrix is characterized by the symmetrical structure and will be of complex Toeplitz structure when the array is a ULA or UCA. However, limited degrees of freedom and direction samples bring about the nonsymmetry and non-Toeplitz characters of the compensation matrix.

Once the compensation matrix is obtained, it can be employed to compensate for the mutual coupling of the array. Assume that the sample matrix for received signals is $\mathbf{X}^{\prime}$ which includes the noise; the calibrated sample matrix can be calculated by the following formula:

$$
\mathbf{X}=\mathbf{C X}^{\prime}
$$

After being calibrated by the compensation matrix, the received signals can be imported to adaptive array processing algorithms. In order to verify the performance of the element pattern reconstruction method, many DOA estimation algorithms can be employed, such as the multiple signal classification (MUSIC), the estimations of signal parameters via rotational invariance techniques (ESPRIT), the maximum likelihood (ML), and the subspace fitting (SF) [20-23].

For an actual antenna array, the received signals influenced by the mutual coupling effect can be generated via the electric field matrix $\mathbf{E}$ which acts as the actual direction matrix. Another approach to obtain the actual direction matrix is to calculate the induced terminal voltage vector of the array due to the incident plane waves in the same directions with the incident signals. Actually, two approaches are equivalent.

2.2. Wideband Mutual Coupling Compensation. In some wideband adaptive systems such as the frequency-hopping system, mutual coupling compensation is carried out for narrowband frequencies, although the entire operating frequency band of the system is of wideband. In this case, the compensation matrix at any frequency is needed. However, it is difficult and unnecessary to store the compensation matrices for all frequencies owing to the limited memory space of the hardware. For the wideband system, each entry of the wideband compensation matrix would vary against the frequency. However, it is difficult to obtain an analytical expression for the mathematical relation between the entries of wideband compensation matrix at different frequencies from a pure electromagnetic theory consideration [15]. From the point of system identification method, approximate expressions can be utilized to represent the entries of wideband compensation matrix [16]. Assume that $\widetilde{c}_{n m}(j k)$ is an approximate expression of the entry $c_{n m}(j k)$; one obtains

$$
\tilde{c}_{n m}(j k)=\frac{A_{0}^{n m}+A_{1}^{n m}(j k)+A_{2}^{n m}(j k)^{2}+A_{3}^{n m}(j k)^{3}+\cdots}{B_{0}^{n m}+B_{1}^{n m}(j k)+B_{2}^{n m}(j k)^{2}+B_{3}^{n m}(j k)^{3}+\cdots},
$$

where $k=\omega \sqrt{\mu_{0} \varepsilon_{0}}$ denotes the wave number or the propagation constant, and the polynomial coefficients in (7) will determine the curve corresponding to $\widetilde{c}_{n m}$ in the frequency band. In order to calculate the polynomial coefficients, complex-curve fitting method proposed by Levy is an effective method that can be employed [17]. Different from what is proposed by Levy, the variable involved in (7) is $j k$ rather than $j \omega$, which can avoid too small polynomial coefficients and provide us with better computational accuracy of the entries. For convenience, (7) can be written by

$$
\begin{aligned}
\tilde{c}_{n m}(j k)=( & \left(A_{0}^{n m}-A_{2}^{n m} k^{2}+A_{4}^{n m} k^{4}-\cdots\right) \\
& \left.+j k\left(A_{1}^{n m}-A_{3}^{n m} k^{2}+A_{5}^{n m}\right) k^{4}-\cdots\right) \\
& \times\left(\left(B_{0}^{n m}-B_{2}^{n m} k^{2}+B_{4}^{n m} k^{4}-\cdots\right)\right. \\
& \left.\quad+j k\left(B_{1}^{n m}-B_{3}^{n m} k^{2}+B_{5}^{n m} k^{4}-\cdots\right)\right)^{-1 / 2} .
\end{aligned}
$$

Except for the calculation of polynomial coefficients, the polynomial orders for each entry are also to be determined. 
Assume that the polynomial orders of the numerator and denominator are denoted as $p$ and $q$, respectively. The calculation of polynomial coefficients

$$
\Theta=\left[A_{0}^{n m}, A_{1}^{n m}, \ldots, A_{p}^{n m} ; B_{0}^{n m}, B_{1}^{n m}, \ldots, B_{q}^{n m}\right]
$$

can be expressed by the following optimization problem [16]:

$$
\begin{gathered}
\widehat{\Theta}=\arg \min _{\Theta} D(\Theta), \\
D(\Theta)=\sum_{i=1}^{N}\left|c_{n m}\left(j \omega_{i} \sqrt{\mu_{0} \varepsilon_{0}}\right)-\widetilde{c}_{n m}\left(j \omega_{i} \sqrt{\mu_{0} \varepsilon_{0}}\right)\right|^{2},
\end{gathered}
$$

where $D(\Theta)$ is calculated in all sampled frequencies of the bandwidth. In order to obtain an accurate wideband compensation matrix, the compensation matrices at sufficient numbers of frequencies need to be calculated by the element pattern reconstruction method. The complex-curve fitting method is thus employed to fit each entry of the wideband compensation matrix. Entries of the wideband compensation matrix vary slowly with the frequency. Therefore, it is convenient to try the polynomial orders with the beginning of the lowest order and then gradually increase the orders until the fitting error gets negligibly small. For simplicity, the polynomial orders of the numerator and denominator are set equal. In this case, the above optimization problem can be carried out by the procedure shown in Figure 1.

As mentioned above, the compensation matrix obtained by the element pattern reconstruction method is not characterized rigorously by the symmetry and complex Toeplitz structure. Therefore, all entries of the compensation matrix are involved in the complex-curve fitting in order to get more accurate compensation effect in the wideband systems. Once the above process is completed, the compensation matrix at any frequency included in the frequency band can be calculated via (8) with a little effort, and only a few polynomial coefficients that describe the curves need to be stored. For the wideband frequency-hopping adaptive array, it is convenient to obtain the compensation matrix for the required frequencies via (8).

The proposed wideband compensation method can be also employed in the DOA estimation of wideband signals $[20,24]$. In this case, DOA estimation method in the frequency domain can be used for wideband signals. The wideband signals are decomposed into multiple narrowband components which are calibrated at the corresponding subbands using the compensation matrices pertaining to these subbands. The DOA estimation is thus carried out by using the MUSIC algorithm at each subband [20]. Finally, the spatial spectrum for the wideband signals is obtained by averaging all the subband spatial spectra over the whole bandwidth. In general, the noise involved in MUSIC algorithms is white Gaussian noise (AWGN) and would become colored after being multiplied by the compensation matrix. However, the compensation matrix is of diagonal dominance and full rank, which would result in limited influence on the performance of MUSIC algorithms.

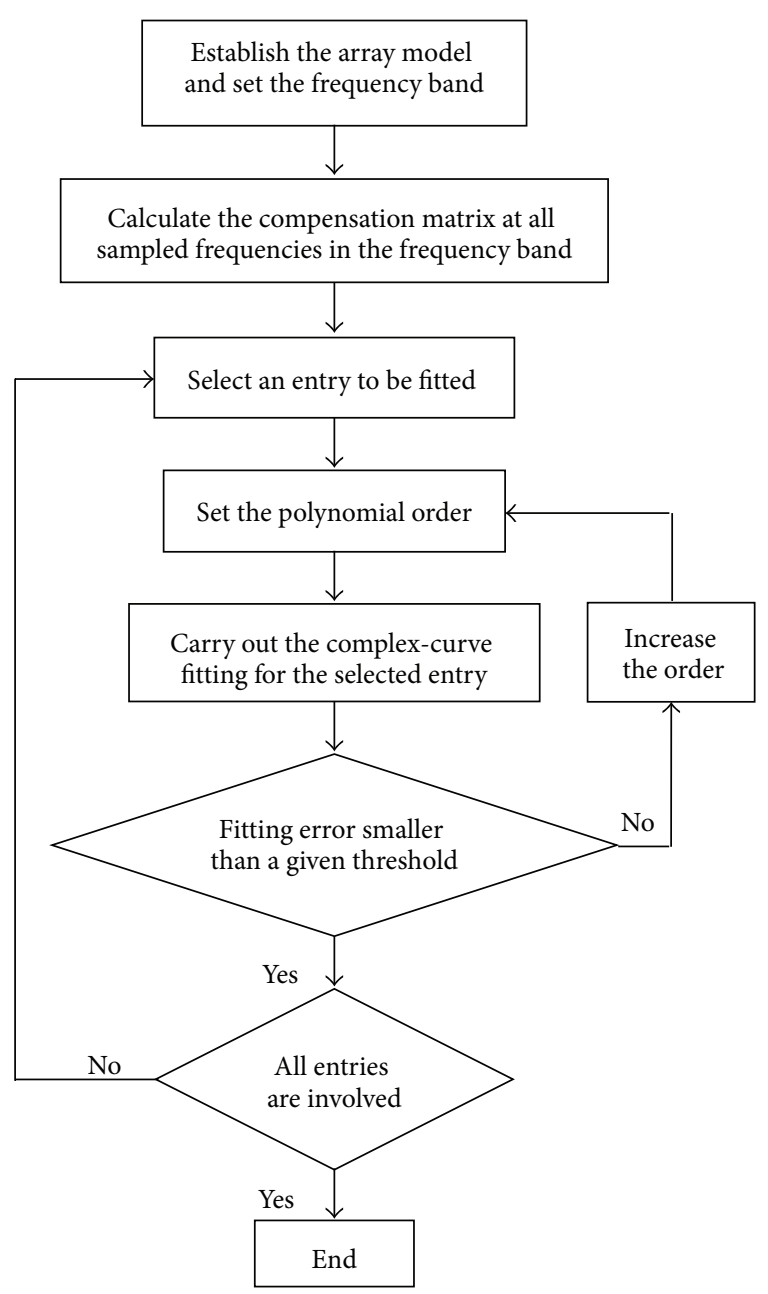

FIGURE 1: Flowchart for the calculation of wideband compensation matrix.

It should be noted that the new wideband compensation method is characterized by the good adaptability of element structures and polarizations owing to the performance of the element pattern reconstruction method. It can be used not only for the dipole or monopole arrays, but also for proper wideband adaptive arrays with wideband microstrip elements or other elements with wideband characteristics.

\section{Numerical Examples}

In this section, a wideband microstrip array is designed to verify the wideband compensation method proposed in this paper. As is shown in Figure 2, the array is composed of five wideband microstrip elements. The VSWR curve of the element is shown in Figure 3. The frequency band of the isolated element is larger than $500 \mathrm{MHz}$ with VSWR $\leq$ 1.5. For the microstrip array, however, the frequency band under consideration is $300 \mathrm{MHz}$ with the center frequency of $2.65 \mathrm{GHz}$. The $3 \mathrm{~dB}$ beamwidth at $2.65 \mathrm{GHz}$ of the element is about $90^{\circ}$. The element spacing of the array is $54 \mathrm{~mm}$, that is, $0.477 \lambda_{0}$, where $\lambda_{0}$ is the wavelength at $2.65 \mathrm{GHz}$. EM simulation tool HFSS Version 13 is utilized to calculate the 


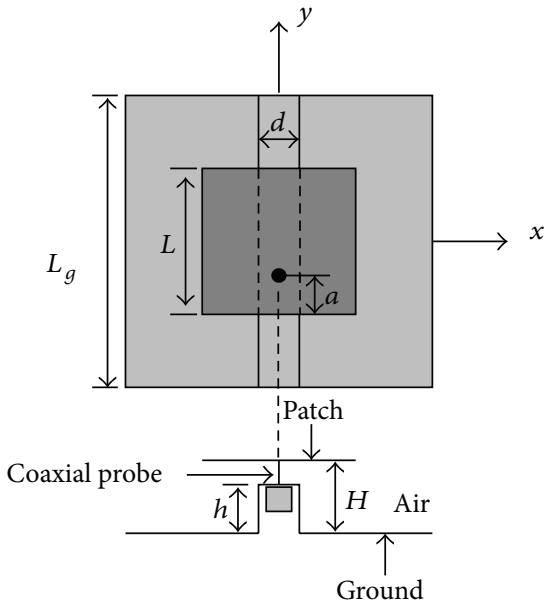

(a)

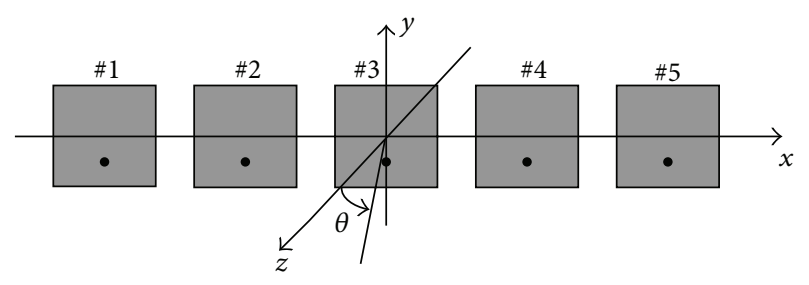

(b)

FIGURE 2: Microstrip element profile (a) and array structures (b). $L_{g}=90 \mathrm{~mm}, d=8 \mathrm{~mm}, a=12 \mathrm{~mm}, L=50 \mathrm{~mm}, H=15 \mathrm{~mm}$, and $h=12 \mathrm{~mm}$.

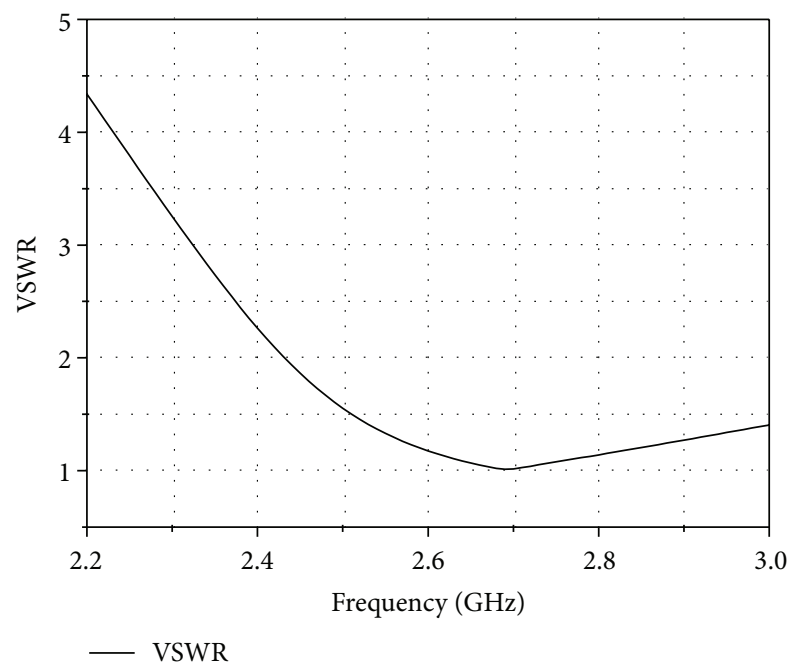

FIgURE 3: VSWR curve of the element in the isolated state.

electric fields of the elements and the array. In the following simulations of DOA estimations, all incident signals are uncorrelated and impinge upon the array from xoz plane. MUSIC algorithm is employed, SNR for all incident signals is $20 \mathrm{~dB}$, and the data sample is 3000 .

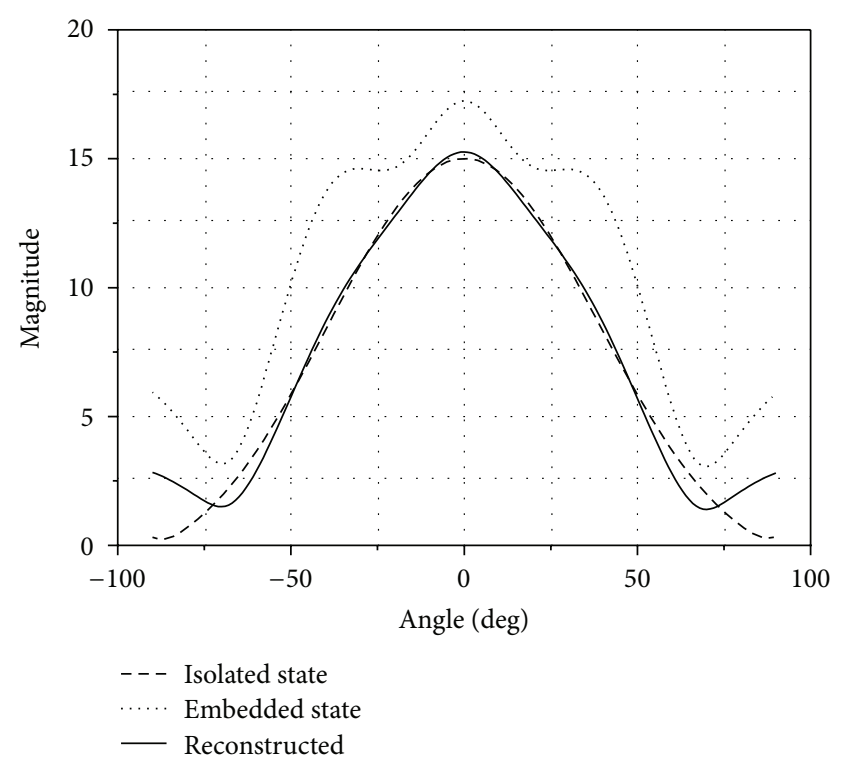

(a)

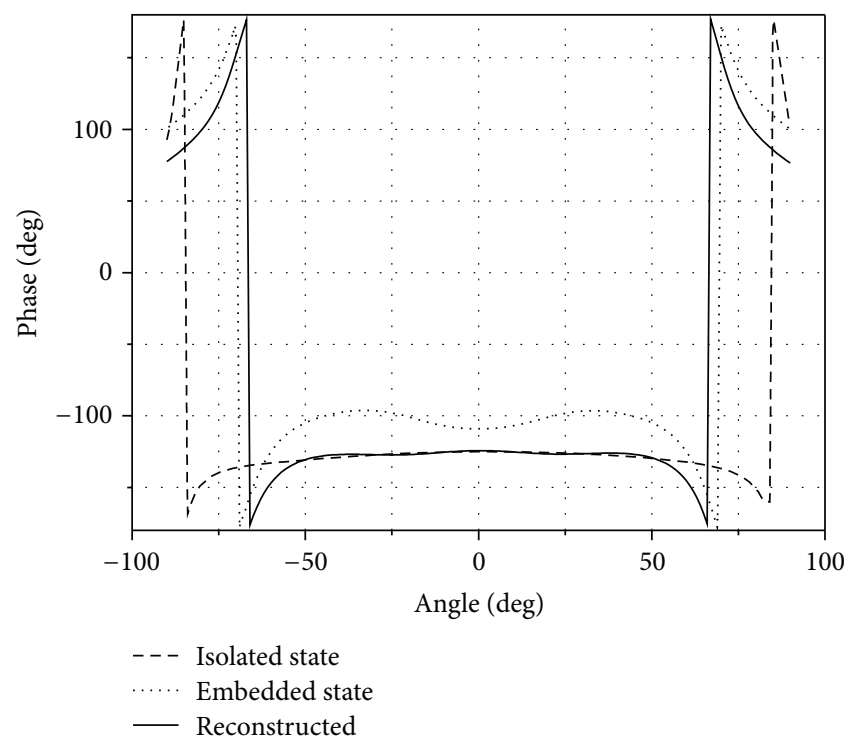

(b)

Figure 4: Pattern reconstruction of $E_{\varphi}$ for element number 3 at $2.65 \mathrm{GHz}$, (a) magnitude and (b) phase.

3.1. Mutual Coupling Compensation Using Element Pattern Reconstruction Method. The element patterns are reconstructed by the compensation matrix obtained via the element pattern reconstruction method. For the linearly polarized microstrip array, the main polarization component is the $\varphi$ component of the electric field in $x o z$ plane which is plane of interest. Therefore, the $\varphi$ component of the electric field is employed to obtain the compensation matrix. In order to reconstruct the element patterns in a larger angle range, 121 directions in the angle range of $\left[-60^{\circ}, 60^{\circ}\right]$ in $x o z$ plane are sampled to calculate the compensation matrix. As an example, the pattern reconstruction for element number 3 at $2.65 \mathrm{GHz}$ is given in Figure 4. It can be seen that 


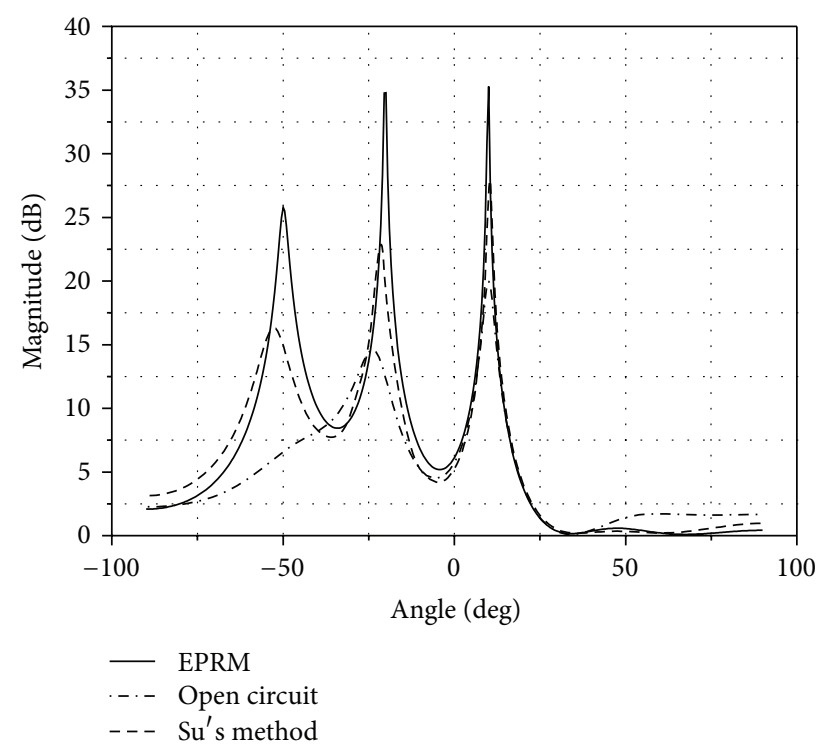

FIGURE 5: Spatial spectrum of the MUSIC algorithm for three incident signals.

the magnitude and phase of the reconstructed pattern are consistent with those of the isolated element pattern in the angle range of $\left[-50^{\circ}, 50^{\circ}\right]$. The angle range of consistence is beyond the $3 \mathrm{~dB}$ beamwidth. It means that the incident signals coming from this angle range will be suitably calibrated by the compensation matrix.

DOA estimations for three incident signals are employed to further verify the effectiveness of the element pattern reconstruction method. The array operates at $2.65 \mathrm{GHz}$, and the compensation matrix obtained above is utilized to calibrate the received signals. Three uncorrelated signals are incident from $\theta=-50^{\circ},-20^{\circ}$, and $10^{\circ}$ in $x o z$ plane, respectively. As shown in Figure 5, the element pattern reconstruction method can provide more accurate DOA estimations in contrast with the open circuit voltage method and Su's method, and DOA estimations for all signals are resolved using the element pattern reconstruction method (EPRM) in Figure 5.

3.2. Calculation of the Wideband Compensation Matrix. In order to implement the optimization procedure to obtain the wideband compensation matrix, 31 compensation matrices with the frequency interval of $10 \mathrm{MHz}$ are calculated using the element pattern reconstruction method. A total of 121 directions in the angle range of $\left[-60^{\circ}, 60^{\circ}\right] x o z$ plane are sampled to calculate each compensation matrix. Complexcurve fitting under given polynomial orders is carried out for each entry of the calibration matrix. Following the process as shown in Figure 1, proper polynomial orders and coefficients are obtained. The polynomial orders for all entries are listed in Table 1. Analytical expressions for all entries are given in the Appendix of this paper. The magnitudes and phases of the entries including $c_{11}, c_{21}, c_{31}, c_{41}$, and $c_{51}$ are shown in Figures 6(a) and 6(b), respectively. It can be seen that the magnitude and phase of each entry obtained through complex-curve

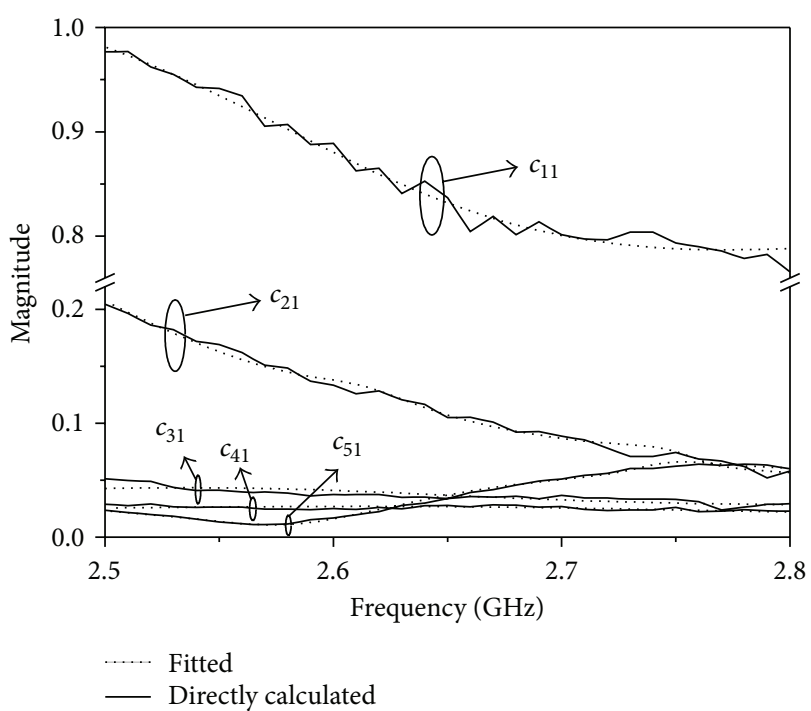

(a)

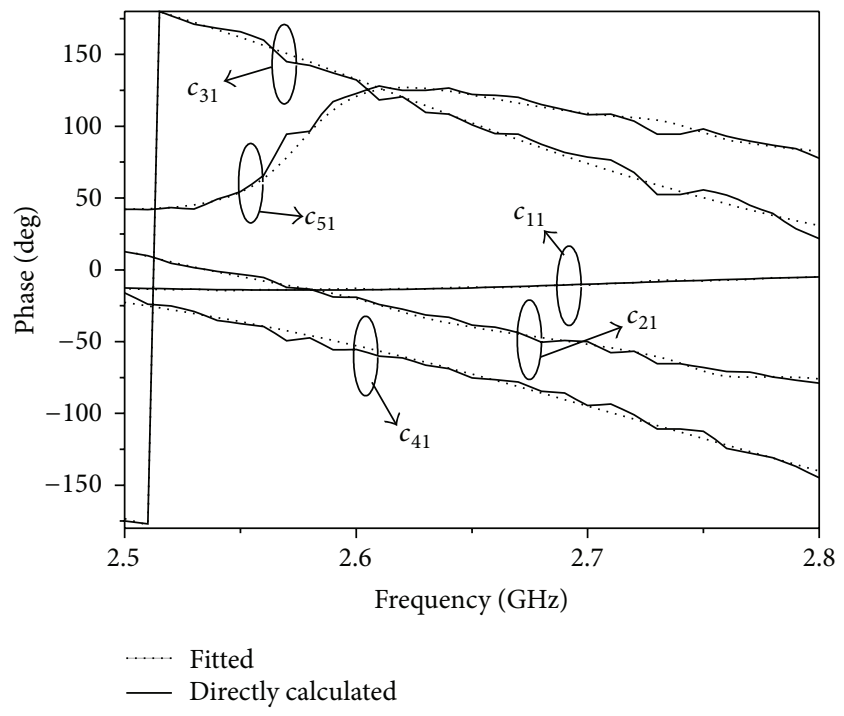

(b)

FIgURE 6: Complex-curve fitting for $c_{11}, c_{21}, c_{31}, c_{41}$, and $c_{51}$, (a) magnitude and (b) phase.

TABle 1: Polynomial orders for all entries of the compensation matrix.

\begin{tabular}{ll}
\hline Polynomial order & Entries of calibration matrix \\
\hline 3 & $c_{11}, c_{23}, c_{31}, c_{32}, c_{34}, c_{41}, c_{43}, c_{44}, c_{55}$ \\
4 & $c_{12}, c_{13}, c_{24}, c_{33}, c_{35}, c_{53}, c_{54}$ \\
5 & $c_{42}, c_{51}$ \\
6 & $c_{21}, c_{22}, c_{52}$ \\
7 & $c_{14}, c_{15}, c_{25}, c_{45}$ \\
\hline
\end{tabular}

fitting agree well in the frequency band with those due to direct calculation via the element pattern reconstruction method, respectively. 


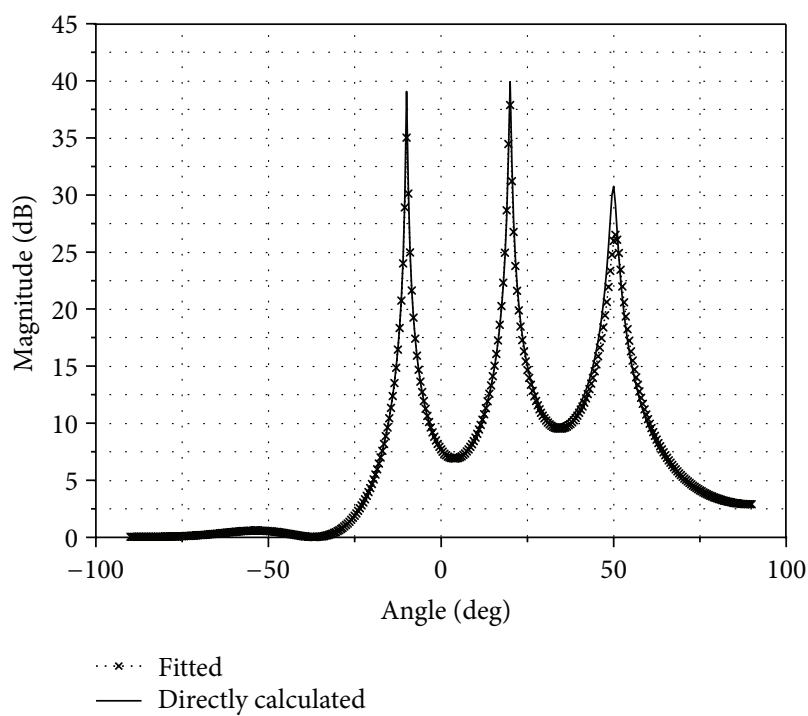

(a)

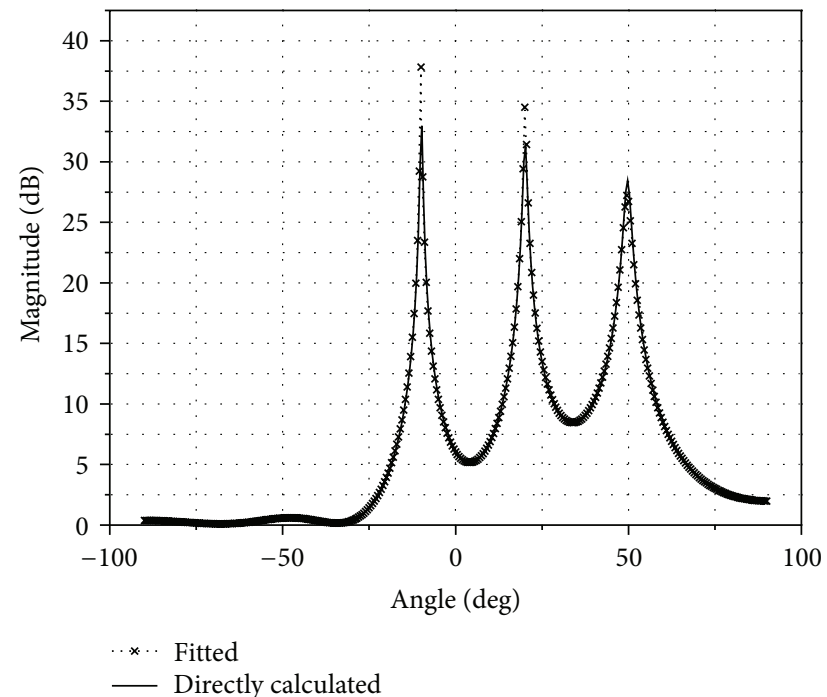

(b)

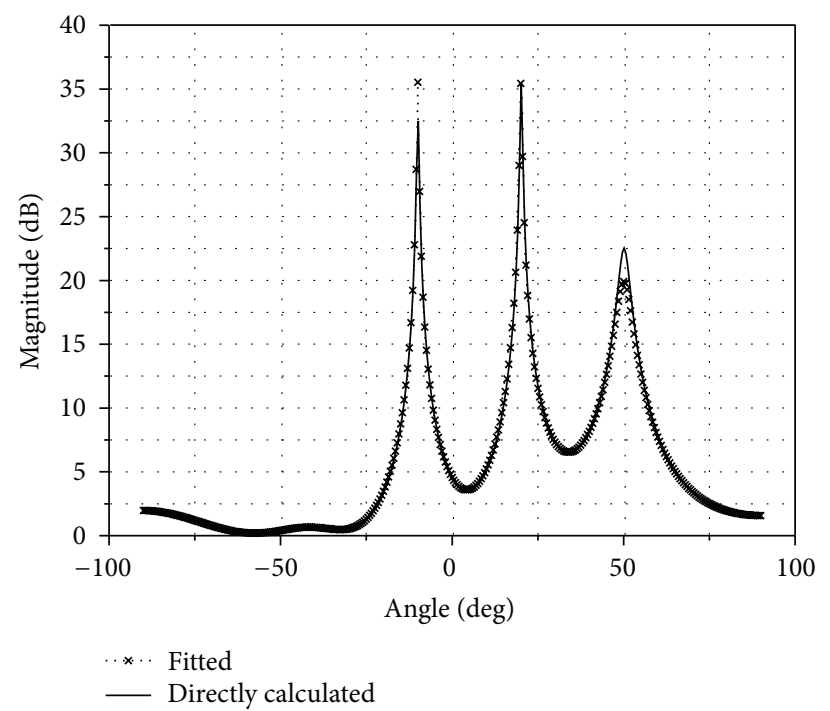

(c)

FIGURE 7: Comparison of DOA estimations due to the directly calculated compensation matrices via element pattern reconstruction method and the fitted compensation matrices via complex-curve fitting at (a) $2.5 \mathrm{GHz}$, (b) $2.65 \mathrm{GHz}$, and (c) $2.8 \mathrm{GHz}$.

For verifying the impact of the fitting error on the mutual coupling compensation, three examples of DOA estimations at $2.5 \mathrm{GHz}, 2.65 \mathrm{GHz}$, and $2.8 \mathrm{GHz}$ obtained from the directly calculated compensation matrices via the element pattern reconstruction method and the fitted compensation matrices via the complex-curve fitting are shown in Figures $7(a), 7(b)$, and $7(\mathrm{c})$. In the DOA estimations, three uncorrelated signals are incident from $\theta=-10^{\circ}, 20^{\circ}$, and $50^{\circ}$, respectively.

From the spatial spectra of MUSIC algorithm at three frequencies, it can be seen that the accuracy of DOA estimations is virtually unattacked by the fitting error. It can be inferred that the complex-curve fitting is robust in the whole frequency band, which would guarantee the real-time calculation of the compensation matrix at any frequency in the frequency band.
3.3. DOA Estimations of the Wideband Signals. The wideband signal under consideration has a bandwidth of $100 \mathrm{MHz}$ covering 2.5 2.6 GHz. DOA estimations of wideband signals are carried out in the narrowband way as described in Section 2. Through the discrete Fourier transform (DFT), the received signal on each element is decomposed into 10 subband signals. The subband signal vectors are calibrated by the compensation matrices owing to the center frequencies of the corresponding subbands. The DOA estimations at each subband are then implemented via MUSIC algorithm. Finally, the average spatial spectrum on the bandwidth provides the DOA estimations of wideband signals.

Spatial spectra of MUSIC algorithm for three wideband signals at all subbands are given in Figure 8, where the compensation matrices via the complex-curve fitting are 


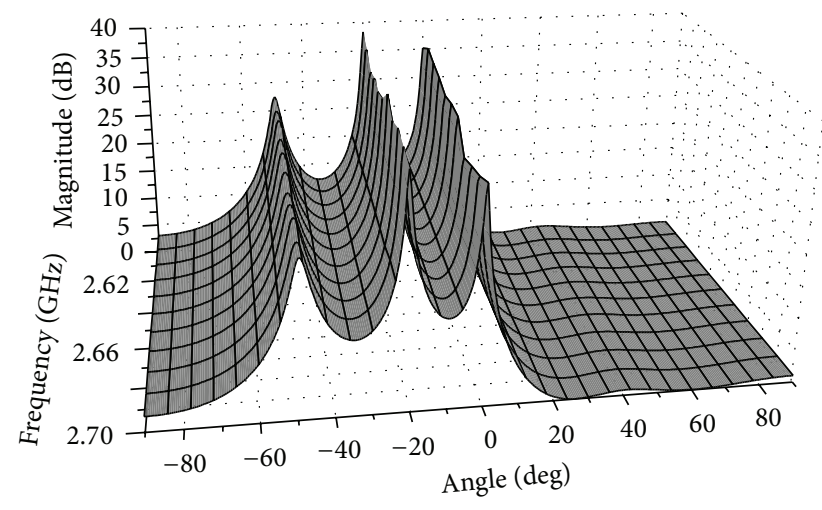

FIGURE 8: Spatial spectra of the MUSIC algorithm for three wideband signals at all subbands. The fitted compensation matrices in the frequency band are employed.

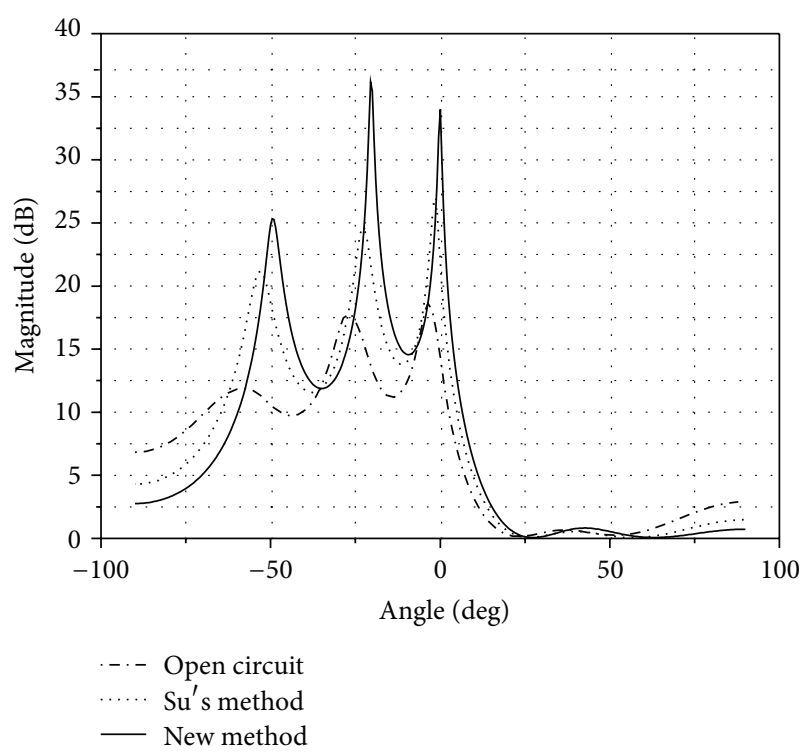

Figure 9: Average spatial spectra due to the new method, the open circuit voltage method, and Su's method.

employed. Three uncorrelated wideband signals are incident from $\theta=0^{\circ},-20^{\circ}$, and $-50^{\circ}$, respectively. Consistence of DOA estimations at various subbands can be seen in Figure 8, which is further indicated by the average spatial spectra as shown in Figure 9. The incident angles of three wideband signals can be found accurately in the average spatial spectra using the proposed wideband compensation method. For comparison, the open circuit voltage method and Su's method are employed. All compensation matrices obtained with the open circuit voltage method and Su's method at sampled frequencies are calculated and directly used in the DOA estimations, respectively. It can be seen that large errors of DOA estimations exist for the open circuit voltage method and Su's method. Estimations bias due to three methods can be found in Table 2 .
TABLE 2: Absolute DOA bias for the wideband signals in three cases.

\begin{tabular}{lcccc}
\hline \multirow{2}{*}{ Incident signal } & \multirow{2}{*}{$\boldsymbol{\circ}\left({ }^{\circ}\right)$} & \multicolumn{3}{c}{ Absolute DOA bias $\left(^{\circ}\right)$} \\
& & New method & Open circuit & Su's method \\
\hline Signal 1 & -50 & 0.7 & 7.8 & 3.0 \\
Signal 2 & -20 & 0.3 & 7.2 & 2.7 \\
Signal 3 & 0 & 0.1 & 3.4 & 1.6 \\
\hline
\end{tabular}

\section{Conclusion}

In this paper, a new wideband compensation method for adaptive arrays is proposed. The element pattern reconstruction method and the system identification method are combined to obtain the mutual coupling compensation matrix for the wideband adaptive array. Owing to the performance of the element pattern reconstruction method, the new wideband compensation method is characterized by the good adaptability of element structures and polarizations. The compensation matrix at any frequency in the frequency band can be obtained conveniently through the analytical expressions of all entries, which benefits wideband mutual coupling compensation. The effectiveness of the proposed wideband mutual coupling compensation method is verified by DOA estimations using a wideband microstrip array.

\section{Conflict of Interests}

The authors declare that there is no conflict of interests concerning the publication of this paper.

\section{Acknowledgments}

This work was supported in part by the Fundamental Research Funds for the Central Universities (K5051202012) and the National Natural Science Foundation of China (61201019).

\section{References}

[1] I. J. Gupta and A. A. Ksienski, "Effect of mutual coupling on the performance of adaptive arrays," IEEE Transactions on Antennas and Propagation, vol. 31, no. 5, pp. 785-791, 1983.

[2] H. T. Hui, "Improved compensation for the mutual coupling effect in a dipole array for direction finding," IEEE Transactions on Antennas and Propagation, vol. 51, no. 9, pp. 2498-2503, 2003.

[3] H. T. Hui, "A new definition of mutual impedance for application in dipole receiving antenna arrays," IEEE Antennas and Wireless Propagation Letters, vol. 3, no. 1, pp. 364-367, 2004.

[4] C. K. Edwin Lau, R. S. Adve, and T. K. Sarkar, "Minimum norm mutual coupling compensation with applications in direction of arrival estimation," IEEE Transactions on Antennas and Propagation, vol. 52, no. 8, pp. 2034-2041, 2004.

[5] T. Su, K. Dandekar, and H. Ling, "Simulation of mutual coupling effect in circular arrays for direction-finding application," Microwave and Optical Technology Letters, vol. 26, no. 5, pp. 331336, 2000.

[6] K. R. Dandekar, H. Ling, and G. Xu, "Experimental study of mutual coupling compensation in smart antenna applications," 
IEEE Transactions on Wireless Communications, vol. 1, no. 3, pp. 480-487, 2002.

[7] R. J. Weber and Y. Huang, "An automatic calibration system for smart antenna arrays," in Proceedings of the IEEE Antennas and Propagation Society International Symposium (APSURSI '09), pp. 1-4, Charleston, SC, USA, June 2009.

[8] Q. Yuan, Q. Chen, and K. Sawaya, "Accurate DOA estimation using array antenna with arbitrary geometry," IEEE Transactions on Antennas and Propagation, vol. 53, no. 4, pp. 1352-1357, 2005.

[9] S. Henault, S. K. Podilchak, S. M. Mikki, and Y. M. M. Antar, "A methodolgy for mutual coupling estimation and compensation in antennas," IEEE Transactions on Antennas and Propagation, vol. 61, no. 3, pp. 1119-1131, 2013.

[10] S. Henault and Y. M. M. Antar, "Limitations of online calibration methods in antenna arrays," in Proceedings of the IEEE Antennas and Propagation Society International Symposium (APSURSI '10), pp. 1-4, Toronto, Canada, July 2010.

[11] S. Henault and Y. M. M. Antar, "Accurate evaluation of mutual coupling for array calibration," in 2009 Computational Electromagnetics International Workshop (CEM '09), pp. 34-37, Izmir, Turkey, July 2009.

[12] B. Friedlander and A. J. Weiss, "Direction finding in the presence of mutual coupling," IEEE Transactions on Antennas and Propagation, pp. 273-284, 1991.

[13] M. Lin and L. Yang, "Blind calibration and DOA estimation with uniform circular arrays in the presence of mutual coupling," IEEE Antennas and Wireless Propagation Letters, vol. 5, no. 1, pp. 315-318, 2006.

[14] F. Sellone and A. Serra, "An iterative algorithm for the compensation of toeplitz mutual coupling in Uniform and linear arrays," in Proceedings of the 12th Digital Signal Processing Workshop, pp. 438-443, Teton National Park, Wyo, USA, September 2006.

[15] B. H. Wang and H. T. Hui, "Wideband mutual coupling compensation for receiving antenna arrays using the system identification method," IET Microwaves, Antennas and Propagation, vol. 5, no. 2, pp. 184-191, 2011.

[16] R. Pintelon, P. Guillaume, Y. Rolain, J. Schoukens, and H. Van hamme, "Parametric identification of transfer functions in the frequency domain - a survey," IEEE Transactions on Automatic Control, vol. 39, no. 11, pp. 2245-2260, 1994.

[17] E. C. Levy, "Complex-curve fitting," IRE Transactions on Automatic Control, pp. 37-43, 1959.

[18] Q. Huang, H. Zhou, J. Bao, and X. Shi, "Accurate DOA estimations using microstrip adaptive arrays in the presence of mutual coupling effect," International Journal of Antennas and Propagation, vol. 2013, Article ID 919545, 8 pages, 2013.

[19] Q. Huang, H. Zhou, J. Bao, and X. Shi, "Accurate calibration of mutual coupling for conformal antenna arrays," Electronic Letters, vol. 49, no. 23, pp. 1418-1420, 2013.

[20] R. O. Schmidt, "Multiple emitter location and signal parameter estimation," IEEE Transactions on Antennas and Propagation, vol. AP-34, no. 3, pp. 276-280, 1986.

[21] R. Roy and T. Kailath, "ESPRIT-estimation of signal parameters via rotational invariance techniques," IEEE Transactions on Acoustics, Speech, and Signal Processing, vol. 37, no. 7, pp. 984995, 1989.

[22] A. G. Jaffer, "Maximum likelihood direction finding of stochastic sources: a separable solution," in Proceedings of the International Conference on Acoustics, Speech, and Signal Processing, vol. 5, pp. 2893-2896, New York, NY, USA, 1988.
[23] J. A. Cadzow, "A high resolution direction-of-arrival algorithm for narrow-band coherent and incoherent sources," IEEE Transactions on Acoustics, Speech, and Signal Processing, vol. 36, no. 7, pp. 965-979, 1988.

[24] M. Wax, T. Shan, and T. Kailath, "Spatio-temporal spectral analysis by eigenstructure methods," IEEE Transactions on Acoustics, Speech, and Signal Processing, vol. 32, no. 4, pp. 817-827, 1984. 

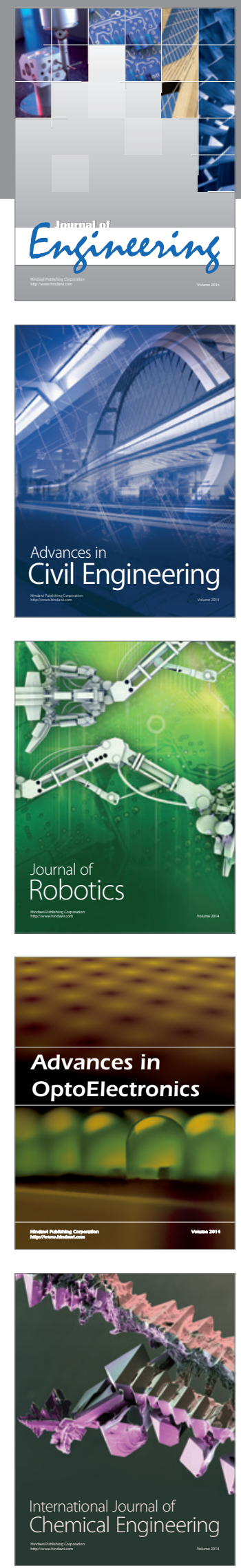

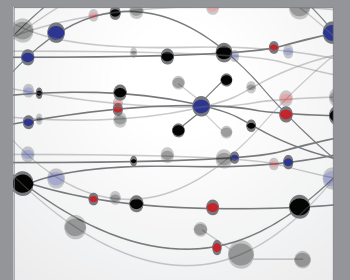

The Scientific World Journal
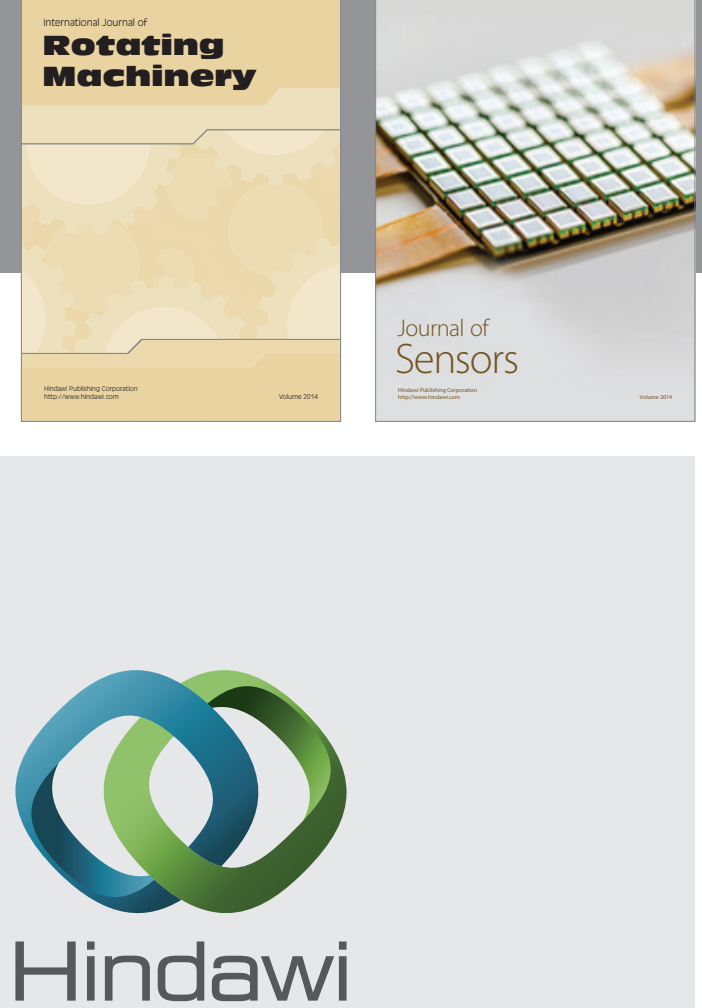

Submit your manuscripts at http://www.hindawi.com
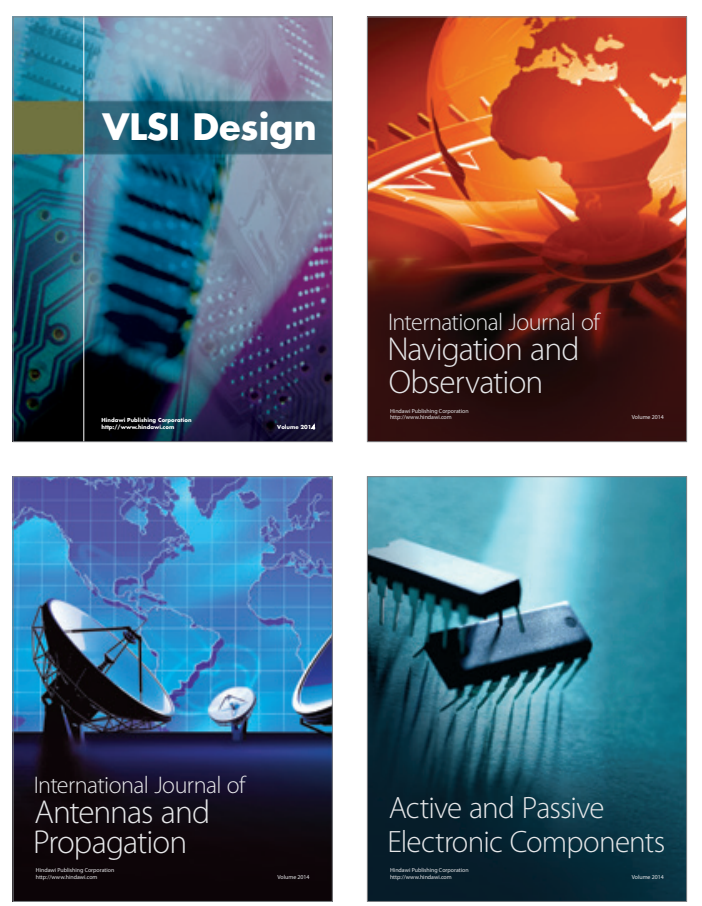
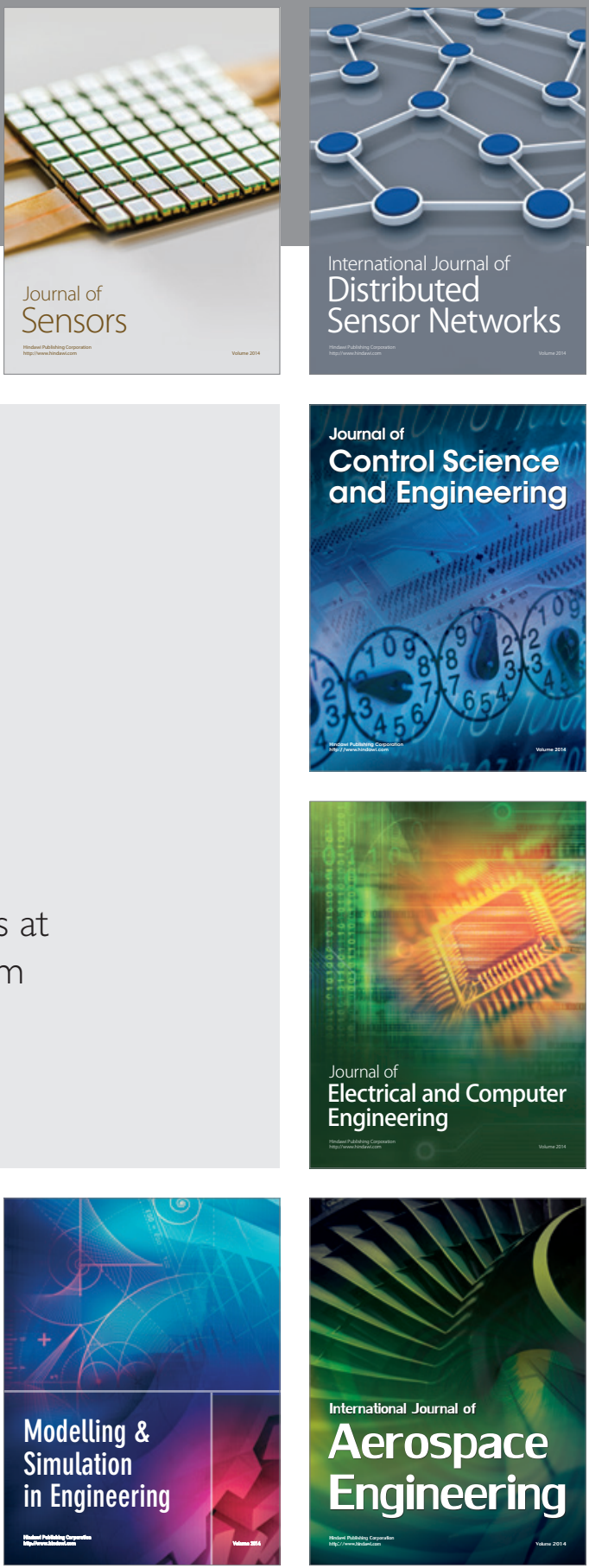

Journal of

Control Science

and Engineering
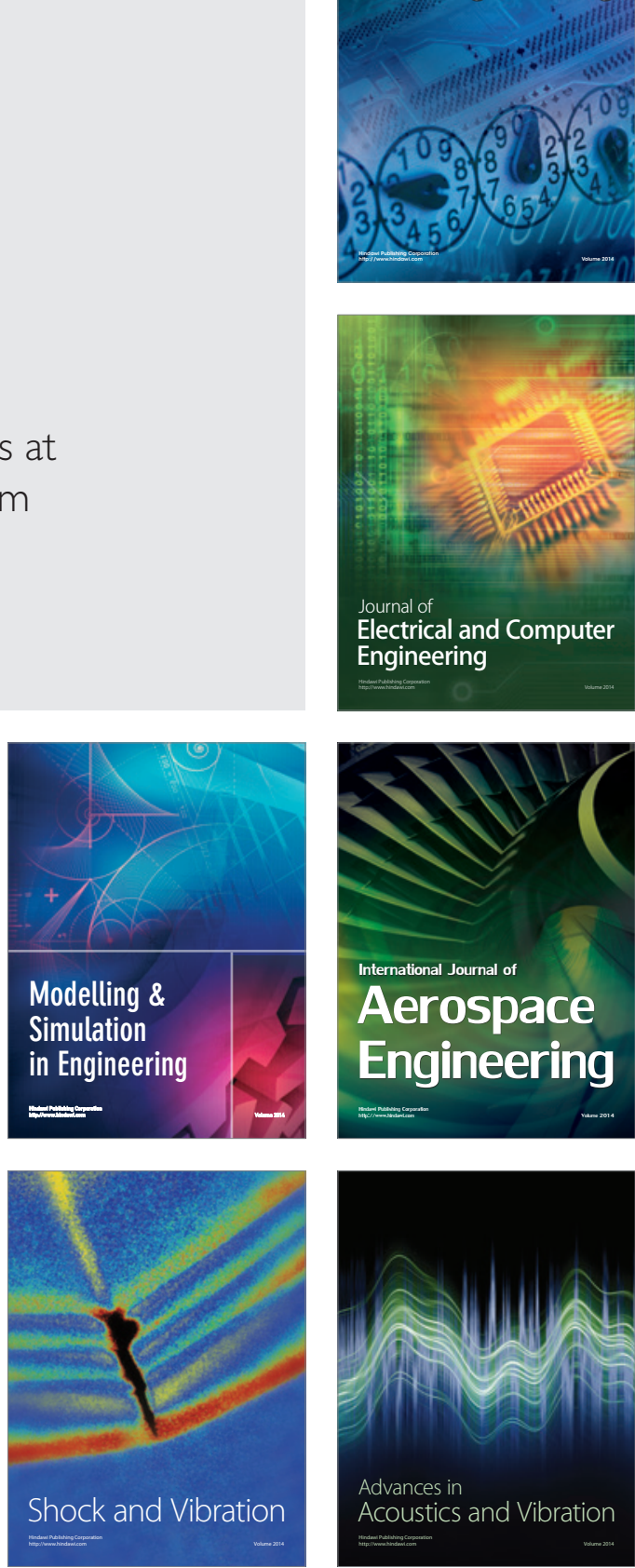